\title{
Diagnostic imaging of gastrointestinal neuroendocrine tumours (GI-NETs): relationship between MDCT features and 2010 WHO classification
}

\author{
Giulia Grazzini ${ }^{1}$ - Ginevra Danti ${ }^{1}$ - Diletta Cozzi ${ }^{1}$ Monica Marina Lanzetta ${ }^{1}$. Gloria Addeo ${ }^{1}$ Massimo Falchini ${ }^{1}$. \\ Antonella Masserelli ${ }^{1} \cdot$ Silvia Pradella ${ }^{1} \cdot$ Vittorio Miele $^{1}[$
}

Received: 29 March 2018 / Accepted: 19 September 2018 / Published online: 25 September 2018

(C) Italian Society of Medical Radiology 2018

\begin{abstract}
Aims We aimed to present our series of gastrointestinal neuroendocrine tumours (GI-NETs) in order to illustrate and highlight the associated contrast-enhanced multi-detector computed tomography (MDCT) features. We also attempted to identify a relationship between MDCT imaging and the 2010 World Health Organization (WHO) classification system.

Materials and methods We selected all patients with pathologically proven GI-NETs diagnosed between January 2010 and August 2017. Only patients undergone contrast-enhanced MDCT imaging in the immediate preoperative period were included in our study. Later, two expert radiologists retrospectively assessed MDCT intestinal and extra-intestinal signs. We also analysed the relationship between MDCT imaging and the 2010 WHO classification.

Results A total of 20 patients (13 males, 7 females, age range 37-89 years, mean age 69.9 years) were included in our study. The majority of GI-NETs (85\%) occurred in the small bowel and mainly in the terminal ileum. Forty-five percentage of our GI-NETs were diagnosed after an access to emergency medical service for obstruction symptoms or gastrointestinal bleeding. Regarding intestinal signs, $15 / 20$ patients showed an intraluminal nodular mass and 5/20 a wall thickening. Extra-intestinal signs were present in $75 \%$ of cases. Desmoplastic reaction and lymph nodes metastases were significantly correlated with higher grade of GI-NETs.

Conclusions The majority of GI-NETs appears as intraluminal mass often associated with extra-intestinal signs. We found a significantly correlation between higher grade of GI-NETs and extra-intestinal signs. MDCT imaging may be useful in predicting the pathological classification of GI-NETs.
\end{abstract}

Keywords Gastrointestinal neuroendocrine tumours $\cdot$ Multi-detector computed tomography $\cdot$ Intestinal signs $\cdot$ Extraintestinal signs $\cdot$ Pathological classification

Vittorio Miele

vmiele@sirm.org

Giulia Grazzini

grazzini.giulia@gmail.com

Ginevra Danti

ginevra.danti@gmail.com

Diletta Cozzi

dilec89@hotmail.it

Monica Marina Lanzetta

monica.lanzetta@virgilio.it

Gloria Addeo

gloria.addeo@gmail.com
Massimo Falchini

massimo.falchini@unifi.it

Antonella Masserelli

antonella.masserelli@tiscali.it

Silvia Pradella

pradella3@yahoo.it

1 Department of Radiology, Azienda OspedalieroUniversitaria Careggi, Largo Brambilla 3, 50134 Florence, Italy 


\section{Introduction}

Gastrointestinal (GI) neuroendocrine tumours (GI-NETs) are rare, accounting for only $2 \%$ of all gastrointestinal tumours [1]. Contrast-enhanced multi-detector computed tomography (MDCT) is the most largely used imaging modality for the localization of GI-NETs. Contrast-enhanced MDCT has allowed an optimization of scan protocols, with many advantages, including (1) very rapid scan times reducing movement artefacts; (2) contrast-enhanced images with arterial and portal phase and (3) the ability to reformat the images in thinner slices, in order to improving resolution and allowing the images to be viewed optimally in different anatomical planes. For these reasons, contrast-enhanced MDCT is the main imaging modality for diagnosis, staging, preoperative evaluation and post-treatment follow-up of NET [2-4]. MDCT images of GI-NETs usually appear as a hyper-enhancing nodular mass arising from the bowel wall or as a regional uniform bowel wall thickening $[4,5]$. The World Health Organization (WHO) classification system of 2010 attempted to grade GI-NETs on the basis of their $\mathrm{Ki}-67$ index and mitotic count, dividing these tumours into well-differentiated neuroendocrine tumours (grades 1 and 2, Ki-67 index $<2 \%$ and 3-20\%, respectively), also termed carcinoid tumours, and poorly differentiated neuroendocrine carcinomas (grade 3, Ki-67>20\%) [6-10]. To the best of our knowledge, in the literature isn't reported any correlation between imaging features of GI-NETs and grading of their pathologic classification. In our study, we aimed to present our series of GI-NETs in order to illustrate and highlight the associated MDCT features. We also attempted to identify a relationship between MDCT imaging and the 2010 WHO classification system.

\section{Materials and methods}

\section{Patients}

With a query ("Gastrointestinal" and "neuroendocrine tumours") of the patients' medical records database at the Careggi Hospital of Florence, a radiologist resident selected all patients with pathologically proven GI-NETs diagnosed between January 2010 and August 2017. Only patients undergone contrast-enhanced MDCT imaging in the immediate preoperative period (1 month) were included in our study. Later, two expert radiologists retrospectively analysed MDCT scans of all these patients. Patients' MDCT images were anonymized and de-identified prior to analysis in order to protect patients' privacy. All patients signed a written consent form.

\section{MDCT imaging}

All CT investigations were carried out on a 64-detector helical CT (Somatom Sensation 64, Siemens Healthcare, Germany).

A standard protocol was used: the patients were scanned in supine with craniocaudal breath-hold scans; all cases underwent non-contrast and contrast-enhanced MDCT scanning with a thickness of the scanning slices of 3 or $5 \mathrm{~mm}$. Iodinated contrast medium (Ultravist 300, Bayer Schering, Berlin, Germany) was injected in the antecubital vein at a flow rate of 3-4 mL/s using an automatic injector, immediately followed by a saline flush $(40-50 \mathrm{~mL})$ with a rate of injection of 3-4 mL/s. The dose of the contrast medium was administered according to the patient's body weight [(mL/kg body weight: $80-100 \mathrm{~mL}(<80 \mathrm{~kg})$ or $100-120 \mathrm{~mL}$ $(>80 \mathrm{~kg})]$. Dual-phasic contrast-enhanced images were obtained during the arterial phase (30-35 s after the start of the injection) and portal venous phase (70-75 s after initiation of the injection).

The parameters for both non-contrast and contrastenhanced CT examination were: tube voltage, $120 \mathrm{kV}$; tube current, 200-250 mAs, depending on the patient's size; beam collimation, $64 \times 0.5 \mathrm{~mm}$; and rotation time, $0.4 \mathrm{~s}$.

\section{Imaging analysis}

All GI-NETs were retrospectively assessed for the MDCT intestinal and extra-intestinal signs. Two expert radiologists in consensus made MDCT images evaluation with arbitration by a third radiologist for discordant interpretations. The readers were blinded to the clinical and pathological data of all of the patients.

Among intestinal signs, we evaluated morphological features, classifying the lesions as the following, in accordance with the existing literature $[4,5,11]$ : intraluminal nodular mass arising from the bowel wall and regional uniform bowel wall thickening.

The extra-intestinal signs included mesenteric metastasis surrounded by a desmoplastic reaction, ascites, lymph nodes and liver metastases. In accordance with the existing literature [5, 12], we classified as mesenteric metastasis a mass-like process generally located in the mesenteric fat near to the primary tumour, which may or may not be calcified. The central mass displays soft-tissue spokes radiating into the mesenteric fat towards the small bowel, like bicycle wheel. These signs were classified as the classic desmoplastic reaction that could lead to kinking of the bowel, resulting in bowel obstruction, or in venous ischaemia $[5,12,13]$. 


\section{Pathological classification}

Histopathological classification of GI-NETs was made according to WHO 2010 guidelines [6-10] as: grade 1 (G1), mitotic count $<2$ per 10 high-power fields (HPF) and/ or $\mathrm{Ki}-67 \leq 2 \%$; grade 2 (G2), mitotic count $2-20$ per $10 \mathrm{HPF}$ and/or Ki-67 3-20\%; and grade 3 (G3), mitotic count $>20$ per $10 \mathrm{HPF}$ and/or Ki-67> $20 \%$.

\section{Statistical methods}

GraphPad Prism v7.0 software (GraphPad Software Inc., La Jolla, CA, USA) was used to perform all statistical analyses. The continuous variables were reported as mean \pm standard deviation, while the categorical variables were presented as numbers and percentages. Normal distribution was assessed with the Shapiro-Wilk test. Comparisons between groups were made using $\chi^{2}$ or Fisher's exact test for categorical data. A value of $P<0.05$ was considered significant.

\section{Results}

Thirty-one patients with histological diagnosis of GI-NETs between January 2010 and August 2017 were selected. Among these, eight patients did not undergo contrastenhanced MDCT in the immediate preoperative period and three patients were excluded for poor MDCT image quality.

A total of 20 patients (13 males, 7 females, age range 37-89 years, mean age 69.9 years) were included in our study.

Of the 20 cases, $3(15 \%)$ were duodenal neuroendocrine tumours and 17 (85\%) were small-bowel neuroendocrine tumours, defined as tumours of the jejunum and of the ileum [14]. 9/17 (52.9\%) small-bowel NETs were localized in the terminal ileum, one of whom in the ileocecal valve (Fig. 1). In $20 \%$ of our cases, the tumours were multiple (Figs. 2, 3).
In our series, 9/20 (45\%) patients accessed to emergency medical services for abdominal pain and obstruction symptoms (5/20) or for gastrointestinal bleeding (4/20). Three patients complained weight loss and abdominal pain as their first symptom. Vomiting was present in two cases, and only one patient showed jaundice due to the presence of a periampullary duodenal tumour. Among all 20 patients, three had classic carcinoid syndrome (15\%) with concurrent liver metastases. Finally, in two cases, GI-NETs were discovered incidentally by MDCT imaging studies performed during follow-up of a lung cancer and of a breast cancer, respectively, (Table 1).

\section{MDCT and histopathological findings}

MDCT findings of each patient are shown in Table 2. Regarding intestinal signs, 15/20 (75\%) patients showed an intraluminal nodular mass (Figs. 1, 2, 3, 4) and 5/20 (25\%) a wall thickening (Figs. 5, 6). Extra-intestinal signs were absent in five cases of our series (25\%), all with intraluminal mass. The other 15 patients $(75 \%)$ presented extra-intestinal signs, often more than one. We observed 8/20 (40\%) cases with desmoplastic reaction (Figs. 3, 5, 6), 12/20 (60\%) with lymph nodes (Figs. 4, 5), 9/20 (45\%) with liver metastases (Fig. 3) and 7/20 (35\%) with ascites (Figs. 3, 5).

Based on the WHO 2010 classification, in our series, we found seven cases (35\%) of G1, nine cases (45\%) of G2 and four cases (20\%) of G3 tumours.

\section{Relationship between MDCT findings of GI-NETs and pathologic classification}

As illustrated in Table 3, all G1 tumours showed an intraluminal mass as intestinal sign and none was associated with desmoplastic reaction or ascites. $33.3 \%$ and $50 \%$ of G2 and G3 tumours, respectively, appeared as intestinal wall thickening.
Fig. 1 Intraluminal nodular mass (G2). Axial (a) and coronal (b) contrast-enhanced MDCT images in the arterial phase demonstrate a wellcircumscribed enhancing mass (white arrows) in the ileocecal valve

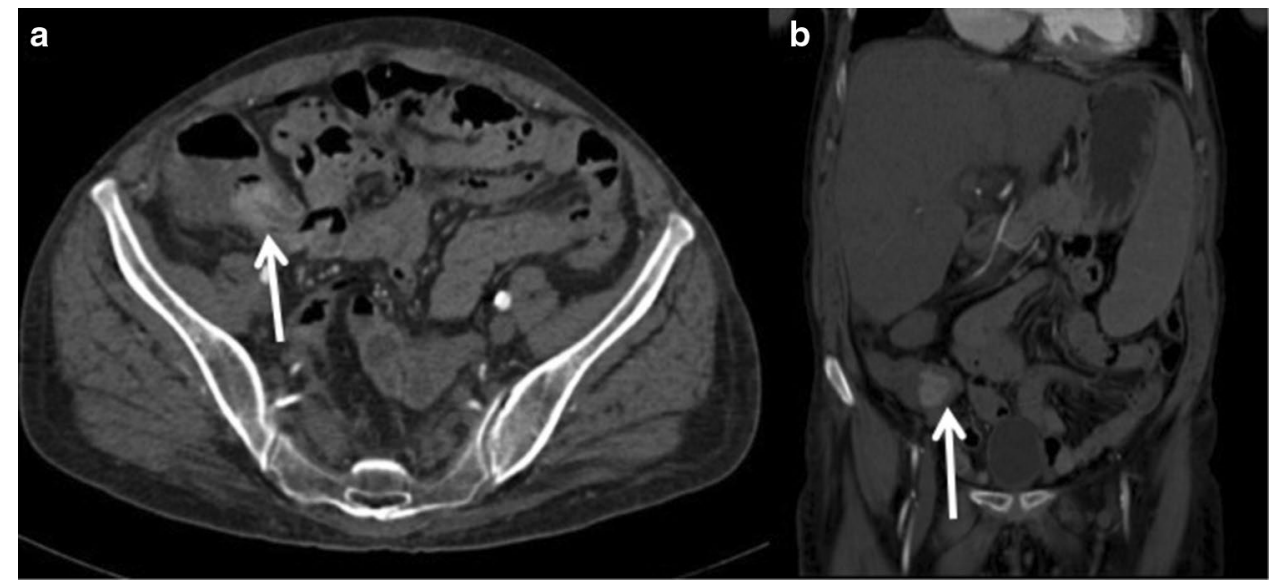


Fig. 2 Multiple non-functioning NETs (G1). Axial (a, b), sagittal (c) and coronal (d) contrastenhanced MDCT images in the arterial phase demonstrate multiple intraluminal masses (white arrows)
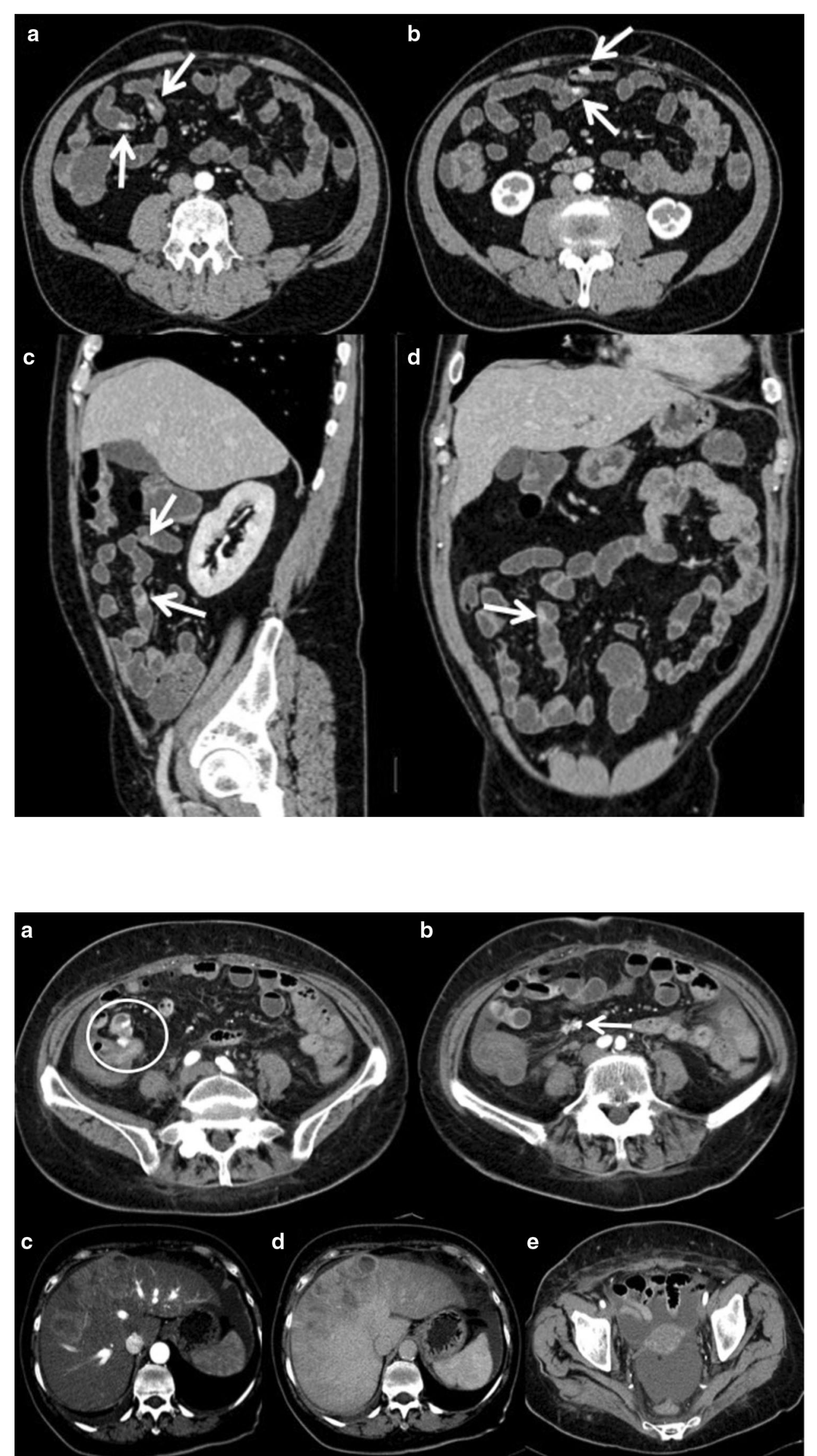

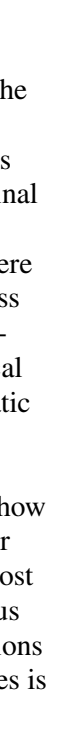

Fig. 3 Multiple functioning NETs (G2). Axial contrastenhanced MDCT image in the arterial phase demonstrates multiple intraluminal masses (a. white circle) in the terminal ileum. In the mesenteric fat near the primary tumour, there is a calcified mesenteric mass (b. white arrow) with radiating soft-tissue strands, typical of mesenteric nodal metastatic disease. Arterial phase (c) and portal venous phase (d) contrast-enhanced MDCT show multiple hyper-vascular liver metastases that become almost isodense on the portal venous phase except for central regions of necrosis. A copious ascites is associated (e) 
Table 1 Demographic data, clinical manifestations and tumour localization of the study population with GI-NETs

\begin{tabular}{|c|c|c|c|c|}
\hline Patients & Sex & Age & Clinical manifestations & GI-NETs location \\
\hline 1. & Male & 84 & Weight loss and abdominal pain & Terminal ileum \\
\hline 2. & Female & 75 & Accessed to emergency medical service for abdominal pain & Ileum \\
\hline 3. & Male & 72 & Weight loss and abdominal pain & Jejunum and ileum (multiple lesions) \\
\hline 4. & Female & 80 & Carcinoid syndrome & Terminal ileum \\
\hline 5. & Female & 72 & Accessed to emergency medical service for abdominal pain & Terminal ileum \\
\hline 6. & Male & 89 & Vomiting & Duodenum \\
\hline 7. & Male & 86 & Accessed to emergency medical service for gastrointestinal bleeding & Jejunum (multiple lesions) \\
\hline 8. & Male & 37 & Vomiting & Jejunum \\
\hline 9. & Male & 56 & Accessed to emergency medical service for gastrointestinal bleeding & Ileum (multiple lesions) \\
\hline 10. & Female & 62 & Carcinoid syndrome & Terminal ileum \\
\hline 11. & Female & 63 & Incidental finding & Terminal ileum \\
\hline 12. & Male & 77 & Weight loss and abdominal pain & Terminal ileum \\
\hline 13. & Male & 72 & Jaundice & Duodenum \\
\hline 14. & Male & 62 & Accessed to emergency medical service for gastrointestinal bleeding & Terminal ileum \\
\hline 15. & Male & 88 & Accessed to emergency medical service for gastrointestinal bleeding & Ileum (multiple lesions) \\
\hline 16. & Female & 77 & Incidental finding & Terminal ileum \\
\hline 17. & Male & 75 & Accessed to emergency medical service for obstruction symptoms & Duodenum \\
\hline 18. & Female & 43 & Accessed to emergency medical service for abdominal pain & Terminal ileum (ileocecal valve) \\
\hline 19. & Male & 46 & Accessed to emergency medical service for obstruction symptoms & Jejunum \\
\hline 20. & Male & 83 & Carcinoid syndrome & Ileum \\
\hline
\end{tabular}

Sex; Age; Clinical manifestations; GI-NETs location, gastrointestinal neuroendocrine tumours location

Table 2 MDCT findings of the study population with pathologically proven GI-NETs

\begin{tabular}{lll}
\hline Patients & Intestinal signs & Extra-intestinal signs \\
\hline 1. & Intraluminal mass & Desmoplastic reaction \\
2. & Intraluminal mass & Lymph nodes \\
3. & Intraluminal mass & Liver metastases \\
4. & Wall thickening & Desmoplastic reaction, lymph nodes, liver metastases, Ascites \\
5. & Intraluminal mass & Desmoplastic reaction, lymph nodes, Ascites \\
6. & Intraluminal mass & Lymph nodes, liver metastases \\
7. & Intraluminal mass & Absent \\
8. & Intraluminal mass & Absent \\
9. & Intraluminal mass & Lymph nodes, liver metastases \\
10. & Wall thickening & Desmoplastic reaction, lymph nodes, liver metastases, ascites \\
11. & Intraluminal mass & Absent \\
12. & Wall thickening & Desmoplastic reaction, lymph nodes \\
13. & Intraluminal mass & Absent \\
14. & Intraluminal mass & Desmoplastic reaction, lymph nodes, liver metastases, ascites \\
15. & Intraluminal mass & Absent \\
16. & Wall thickening & Desmoplastic reaction, lymph nodes, liver metastases, ascites \\
17. & Intraluminal mass & Liver metastases \\
18. & Intraluminal mass & Lymph nodes, liver metastases \\
19. & Intraluminal mass & Desmoplastic reaction, lymph nodes, ascites \\
20. & Wall thickening & Lymph nodes, liver metastases, ascites \\
\hline & &
\end{tabular}

$M D C T$ multi-detector computed tomography, GI-NETs gastrointestinal neuroendocrine tumours 
Fig. 4 Duodenal neuroendocrine tumour (G1). Axial contrast-enhanced MDCT image in the arterial phase demonstrates an intraluminal nodular mass of the duodenum (a. white arrow). Axial contrastenhanced CT image in the portal venous phase shows an enhanced enlarged lymph node (b. black star), suggestive of a metastatic lymph node
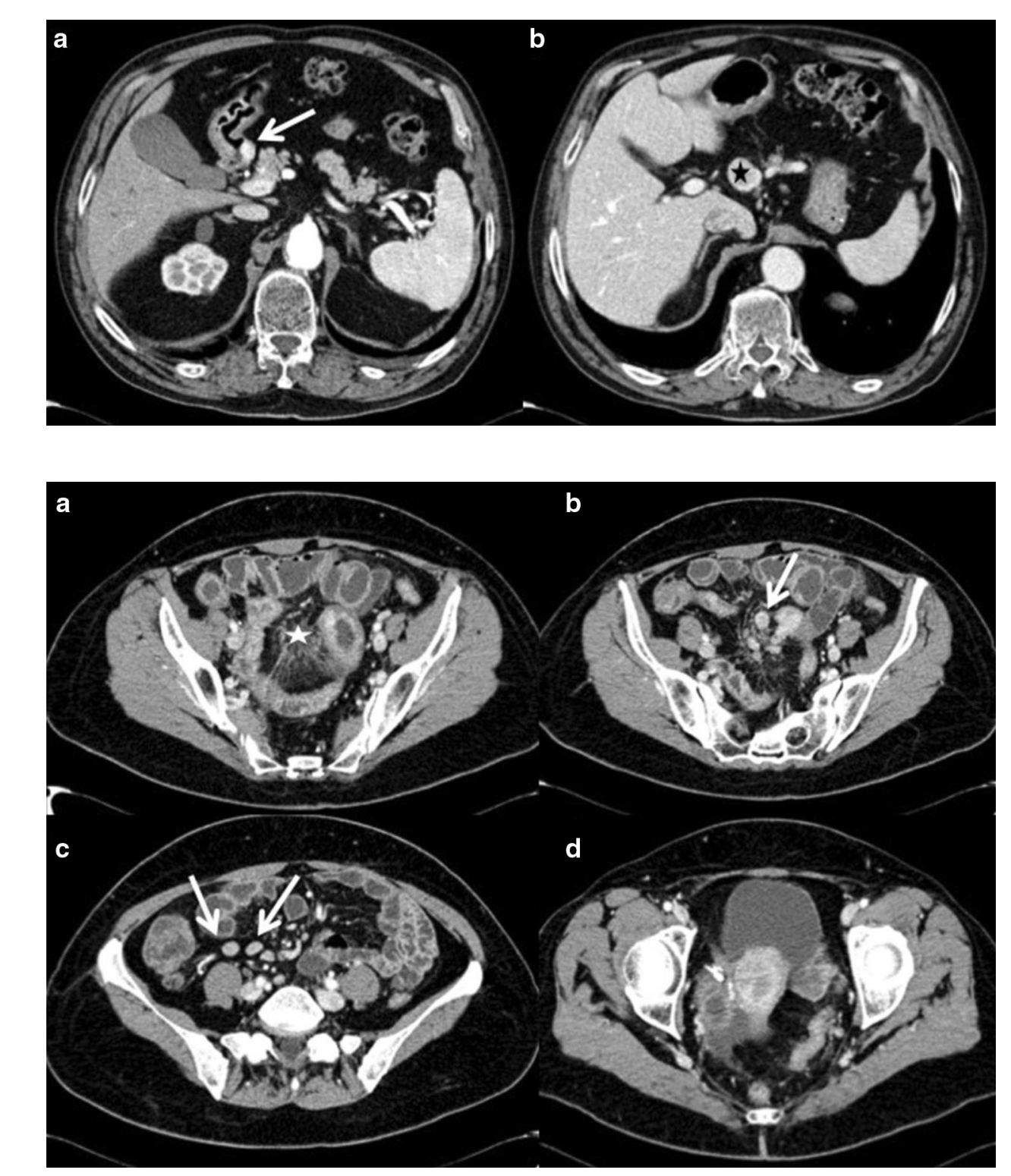

Fig. 5 Gastrointestinal neuroendocrine carcinoma (G3) in the terminal ileum. Axial contrastenhanced MDCT image in the portal venous phase (a) demonstrates a bowel wall thickening of the terminal ileum and a stellate soft-tissue nodule (white star) in the mesentery, typical of a desmoplastic reaction. Just above, there are several lymph nodes metastases (white arrows) (b, c). In the inferior abdomen (d), we can observe ascites

Comparison between G1 and G2-G3 tumours revealed statistically significant differences in desmoplastic reaction and lymph nodes metastases $(P=0.028$ and $P=0.009$, respectively). These extra-intestinal signs were more frequent in high-grade tumours. There were no statistically significant differences in intestinal signs, liver metastases and ascites (Table 3 ).

\section{Discussion}

In the last 20 years, the incidence of GI-NET has increased probably due to recent improvements in diagnostic techniques such as the diffusion of thin-section multi-detector CT. Although NETs can occur in any organ, approximately $60-70 \%$ of these tumours arise in the gastrointestinal tract because it has the largest reservoir of neuroendocrine cells in the body [15]. Studies analysing data from 1973 to 1997 found that the most common site of GI-NETs is the small bowel, defined as jejunum and ileum [16]. Our study confirmed that the majority of GI-NETs $(85 \%)$ occurs in the small bowel and mainly in the terminal ileum.

At the time of initial diagnosis, half of the patients with GI-NETs have advanced disease [17], with metastases to the regional lymph nodes, liver and, finally, bone [18, 19]. In literature, nodal involvement is reported in $41 \%$ of GINETs and distant metastases in 30\% [5]. This is due to the delayed diagnosis of NETs in patients asymptomatic or with non-specific symptoms. In addition, the carcinoid syndrome occurs in the presence of liver metastases because only with hepatic metastatic disease the biologically active metabolite (5-hydroxy-tryptamine), which is otherwise inactivated in 
Fig. 6 Neuroendocrine tumour (G2) in the terminal ileum. Axial (a) and coronal (c) contrast-enhanced MDCT images demonstrate a bowel wall thickening of the terminal ileum (white arrows): the thickened wall is homogeneously enhanced. Just above, there is a stellate soft-tissue nodule (b. white circle) in the mesentery, suggestive of a desmoplastic reaction that leads to bowel angulation

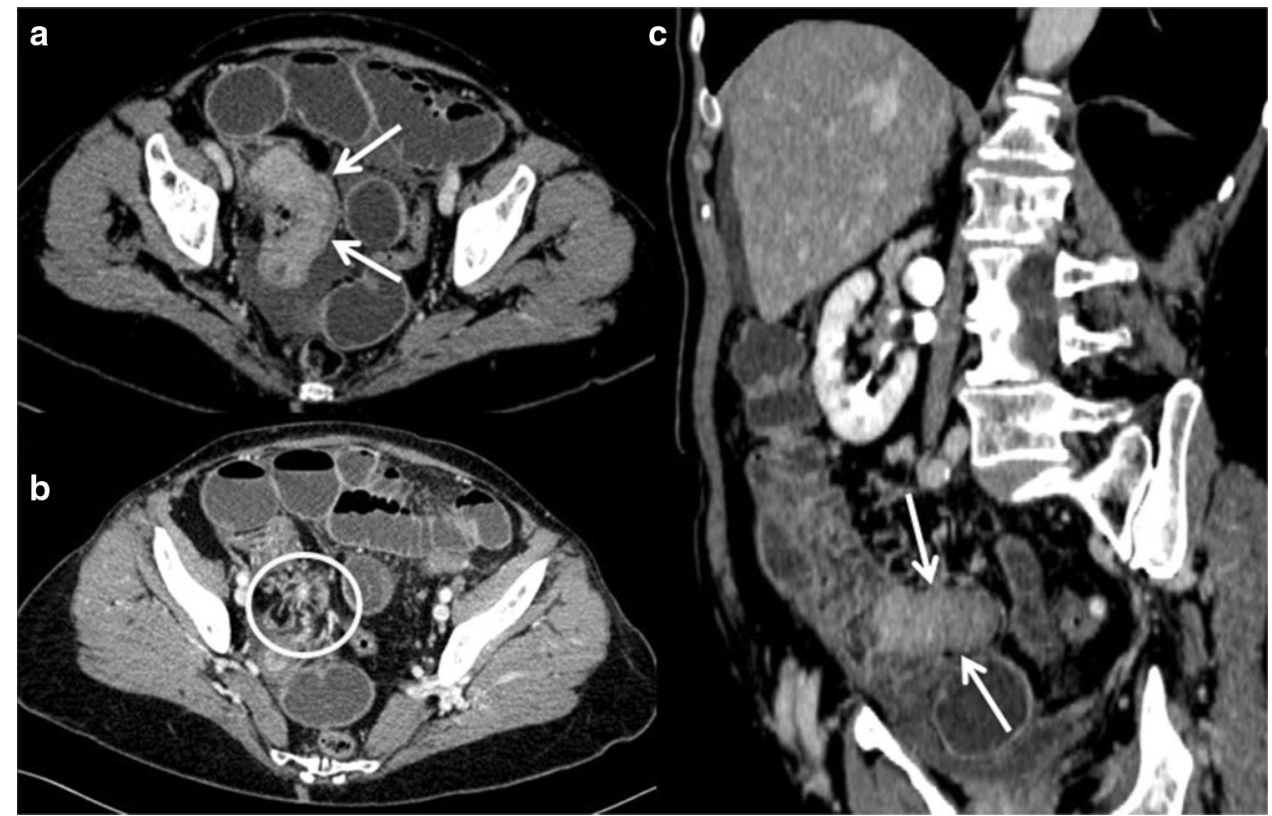

Table 3 Relationship between MDCT findings of GI-NETs and grading system according to the updated WHO classification

\begin{tabular}{|c|c|c|c|c|}
\hline & G1 (7) & G2 (9) & G3 (4) & $P$ \\
\hline \multicolumn{5}{|l|}{ Intestinal signs } \\
\hline Intraluminal mass & $7 / 7(100 \%)$ & $6 / 9(66.6 \%)$ & $2 / 4(50 \%)$ & 0.176 \\
\hline Wall thickening & $0 / 7(0 \%)$ & $3 / 9(33.3 \%)$ & $2 / 4(50 \%)$ & 0.176 \\
\hline \multicolumn{5}{|l|}{ Extra-intestinal signs } \\
\hline Desmoplastic reaction & $0 / 7(0 \%)$ & $6 / 9(66.6 \%)$ & $2 / 4(50 \%)$ & 0.028 \\
\hline Lymph nodes & $1 / 7(14 \%)$ & $8 / 9(88.8 \%)$ & $3 / 4(75 \%)$ & 0.009 \\
\hline Liver metastases & $2 / 7(29 \%)$ & $5 / 9(55.5 \%)$ & $3 / 4(75 \%)$ & 0.348 \\
\hline Ascites & $0 / 7(0 \%)$ & $4 / 9(44.4 \%)$ & $3 / 4(75 \%)$ & 0.055 \\
\hline
\end{tabular}

MDCT multi-detector computed tomography, GI-NETs gastrointestinal neuroendocrine tumours, WHO World Health Organization, G1 grade $1, G 2$ grade $2, G 3$ grade 3

the liver, can reach the systemic circulation [4]. The presence of the characteristic carcinoid syndrome can facilitate NETs detection, otherwise patients present non-specific symptoms, such as weight loss, bleeding or abdominal pain or are asymptomatic [20, 21]. In accordance with the literature, we found only $15 \%$ of our patients with carcinoid syndrome while the others complained non-specific symptoms or are asymptomatic. Forty-five percentage of our GI-NETs were diagnosed after an access to emergency medical services for obstruction symptoms or gastrointestinal bleeding. This result demonstrates how the radiologists should suspect GI-NETs also in the emergency setting.

In MDCT, after administration of contrast medium, the primary tumour usually presents as a hyper-enhancing, nodular mass arising from the bowel wall or as a regional uniform bowel wall thickening [4, 5]. Dohan et al., in their study on the imaging presentation of NETs on magnetic resonance-enterography, found that the most common intestinal signs of NETs were a well-individualized intraluminal mass (84\%) [22]. Similar to their results, we observed intraluminal nodular mass in $75 \%$ of our patients and wall thickening in $25 \%$.

A very typical extra-intestinal finding in GI-NETs is the mesenteric metastasis, which generally appears as a masslike process in the mesenteric fat near the primary tumour. Frequently, this lesion presents calcifications. The mesenteric mass is associated with a surrounding desmoplastic reaction, which is represented by soft-tissue spokes radiating from the central mass towards the small bowel. Frequently, this extra-intestinal finding leads to bowel kinking and angulation. Probably, the desmoplastic reaction is due to the effects of serotonin and other vasoactive peptides produced by the tumour $[5,12]$. In our study, $75 \%$ of GI-NETs had extra-intestinal signs at the time of initial diagnosis. We observed desmoplastic reaction in $40 \%$ of cases, lymph nodes metastases in $60 \%$, liver metastases in $45 \%$ and ascites in $35 \%$.

The WHO 2010 classification system is currently wellaccepted by clinicians as a simple, reproducible, and prognostic effective grading of GI-NETs, since the WHO classification from 2017 is only for pancreatic NET (P-NETs) [6]. The WHO classified GI-NETs into well-differentiated tumours with benign or uncertain behaviour $(\mathrm{G} 1)$; welldifferentiated tumours with low-grade malignant behaviour (G2); and poorly differentiated carcinomas with high-grade malignant behaviour (G3) [6-10]. Among imaging modalities, MDCT plays an important role in the diagnosis and staging of GI-NETs [4]. In recent literature, concerning 
GI-NETs, no correlation between imaging features and grading of pathologic classification is reported. In this study, we evaluated intestinal and extra-intestinal MDCT signs of GI-NETs and attempted to identify any correlation with pathological classification. Our results indicated that MDCT imaging features may be predictive of GI-NETs classifications.

In our series, all G1 tumours appeared as intraluminal mass while $50 \%$ of G3 tumours showed a bowel wall thickening as intestinal sign. Nevertheless, no statistically significant differences were found in intestinal signs.

Comparing MDCT and histopathological findings, we found statistically significant difference among G1, G2 and G3 tumours in desmoplastic reaction and lymph nodes metastases. Indeed, desmoplastic reaction and lymph nodes metastases are significantly correlated with higher grade of GI-NETs. All G2 and G3 tumours had almost one extraintestinal manifestation. This is likely due to the fact that the pathological grading criteria were established based on the mitotic count and $\mathrm{Ki}-67$ index, both of which reflect the proliferation and invasiveness of tumour cells [23, 24].

Several criticisms may be raised with respect to our study. First, this study is retrospective. Second, although we examined GI-NETs between January 2010 and August 2017, our sample size is rather small consisting of 20 patients because patients did not undergo contrast-enhanced MDCT in the immediate preoperative period or with poor MDCT image quality were excluded. Another limitation of this study is that all of our patients were selected because they had histological confirmation of GI-NET after surgical resection. Therefore, it may be argued that this might have introduced a selection bias, because only patients with resectable tumours were included. Thus, it may be possible that the distribution of grading correlation we found is different in a more general population.

In conclusion, as confirmed in the literature, our study showed that the majority of GI-NETs appears as intraluminal nodular mass often associated with extra-intestinal signs on MDCT imaging.

On the other hand, we found a statistically significant correlation between higher grade of GI-NETs and extra-intestinal signs, not previously demonstrated. Therefore, MDCT imaging is a technique that may be useful in predicting the pathological classification of GI-NETs.

\section{Compliance with ethical standards}

Conflict of interest The authors declare that they have no conflict of interest related to the publication of this article. No funding was received for this study.

Ethical approval All procedures performed in the studies involvement human participants were in accordance with the ethical standards of the institutional and/or national research committee and with the 1964
Helsinki declaration and its later amendments or comparable ethical standards. Informed consent was obtained from all individual participants included in the study.

\section{References}

1. Klöppel G, Perren A, Heitz PU (2004) The gastroenteropancreatic neuroendocrine cell system and its tumors: the WHO classification. Ann N Y Acad Sci 1014:13-27

2. Tsai SD, Kawamoto S, Wolfgang CL, Hruban RH, Fishman EK (2015) Duodenal neuroendocrine tumors: retrospective evaluation of CT imaging features and pattern of metastatic disease on dual-phase MDCT with pathologic correlation. Abdom Imaging 40:1121-1130

3. Dahdaleh FS, Lorenzen A, Rajput M et al (2013) The value of preoperative imaging in small bowel neuroendocrine tumors. Ann Surg Oncol 20:1912-1917

4. Rockall AG, Reznek RH (2007) Imaging of neuroendocrine tumours (CT/MR/US). Best Pract Res Clin Endocrinol Metab 21:43-68

5. Ganeshan D, Bhosale P, Yang T, Kundra V (2013) Imaging features of carcinoid tumors of the gastrointestinal tract. AJR Am J Roentgenol 201:773-786

6. Klöppel G (2017) Neuroendocrine neoplasm: dichotomy, origin and classifications. Visc Med 33:324-330

7. Lloyd RV, Osamura RY, Klöppel G, Rosai J (2017) WHO classification of tumours of endocrine organs. IARC Press, Lyon

8. Klimstra DS (2016) Pathologic classification of neuroendocrine neoplasms. Hematol Oncol Clin N Am 30:1-19

9. Rindi G, Petrone G, Inzani F (2014) The 2010 WHO classification of digestive neuroendocrine neoplasms: a critical appraisal four years after its introduction. Endocr Pathol 25:186-192

10. Bosman FT, Carneiro F, Hruban RH, Theise ND (eds) (2010) WHO classification of tumours of the digestive system, vol 3, 4th edn. IARC Press, Lyon

11. Hristova L, Placé V, Nemeth J, Boudiaf M, Laurent V, Soyer P (2012) Small bowel tumors: spectrum of findings on 64-section CT enteroclysis with pathologic correlation. Clin Imaging 36:104-112

12. Sundin A, Arnold R, Baudin E et al (2017) ENETS consensus guidelines for the standards of care in neuroendocrine tumors: radiological, nuclear medicine and hybrid imaging. Neuroendocrinology 105:212-244

13. Tamm EP, Kim EE, Ng CS (2007) Imaging of neuroendocrine tumors. Hematol Oncol Clin N Am 21:409-432

14. Soyer P, Boudiaf M, Fishman EK et al (2011) Imaging of malignant neoplasms of the mesenteric small bowel: new trends and perspectives. Crit Rev Oncol Hematol 80:10-30

15. Taal BG, Visser O (2004) Epidemiology of neuroendocrine tumours. Neuroendocrinology 80(Suppl 1):3-7

16. Maggard MA, O'Connell JB, Ko CY (2004) Updated populationbased review of carcinoid tumors. Ann Surg 240:117-122

17. Yao JC, Hassan M, Phan A et al (2008) One hundred years after "carcinoid": epidemiology of and prognostic factors for neuroendocrine tumors in 35,825 cases in the United States. J Clin Oncol 26:3063-3072

18. Kaltsas GA, Besser GM, Grossman AB (2004) The diagnosis and medical management of advanced neuroendocrine tumors. Endocr Rev 25:458-511

19. Metz DC, Jensen RT (2008) Gastrointestinal neuroendocrine tumors: pancreatic endocrine tumors. Gastroenterology 135:1469-1492 
20. Modlin IM, Kidd M, Latich I, Zikusoka MN, Shapiro MD (2005) Current status of gastrointestinal carcinoids. Gastroenterology 128:1717-1751

21. Oberg KE (2012) The management of neuroendocrine tumours: current and future medical therapy options. Clin Oncol (R Coll Radiol) 24:282-293

22. Dohan A, El Fattach H, Barat M et al (2016) Neuroendocrine tumors of the small bowel: evaluation with MR-enterography. Clin Imaging 40:541-547
23. Rindi G, Arnold R, Bosman F et al (2010) Nomenclature and classification of neuroendocrine neoplasms of the digestive system. In: Bosman F, Carneiro F, Hruban R, Theise N (eds) WHO classification of tumours of the digestive system. IARC Press, Lyon, pp 13-14

24. Zhu H, Ying L, Tang W, Yang X, Sun B (2017) Can MDCT or EUS features predict the histopathological grading scheme of pancreatic neuroendocrine neoplasms? Radiol Med 122:319-326 